\title{
Valoración del rendimiento físico inicial y acciones estratégicas para disminuir la deserción deportiva en el atletismo femenino de la federación deportiva de Loja.
}

Assessment of the initial physical performance and strategic actions to decrease the dropout rate in the athletics sports of the sporting federation of Loja.

Raynier Montoro Bombú. ${ }^{1}$, Edwin Ochoa Granda. ${ }^{2}$ Vladimir Quizhpe Luzuriaga. ${ }^{3} \&$ Luis Valverde Jumbo. ${ }^{4}$

Recibido: 14-06-2017 / Revisado: 17-08-2017 Aceptado: 03-09-2018/ Publicado: 01-10-2018

\begin{abstract}
.
DOI: https://doi.org/10.33262/cienciadigital.v2i4.206

Sports performance seeks to enhance the physical and psychological function as part of a specific sport, and it is implicit in the index of personal and family motivation to prevent sports desertion and thus systematize sports training; therefore, managing the process of sports initiation involves relating variables such as initial physical performance, sports desertion and family ties for the taking of strategic actions. Therefore, the objective of the research is to develop a group of actions that allow stimulating the initiation and permanence of girls identified as talents, for the practice of children's athletics in the Sports Federation of Loja. The research is framed in the characteristics of the description, transversality and correlation. 30 athletes are studied, female sex (8-11 years), and 56 parents of them using the survey technique, also applying the Height, Speed (30m), Long Jump, Abdominals, Irons and Resistance $(400 \mathrm{~m})$. From the description of the individual potentials of the athletes, and of the relatives, 13 strategic actions were designed to enhance the permanence of the athletes

\footnotetext{
${ }^{1}$ Universidad Técnica De Ambato. Carrera de Cultura Física, Ambato, Ecuador. r.montoro@uta.ed.ec

${ }^{2}$ Universidad Nacional de Loja. Carrera de Cultura Física y Deportes. Ecuador, vlady39quizhpe@gmail.com

${ }^{3}$ Universidad Nacional de Loja. Carrera de Cultura Física y Deportes. Ecuador, edwinochoag@hotmail.com

${ }^{4}$ Universidad Nacional de Loja. Carrera de Cultura Física y Deportes. Ecuador, luisrafaelvj@yahoo.es
} 
in the training, allowing to maintain the communication between the family, the girls, the teaching staff and the coaches.

Keywords: Athletics. Bullet Impulsion, Biomechanical Analysis.

\section{Resumen.}

El rendimiento deportivo busca potenciar la función física y psicológica como parte de un deporte determinado, y en él está implícito el índice de motivación personal y familiar para evitar la deserción deportiva y por ende sistematizar el entrenamiento deportivo; por ello, gestionar el proceso de iniciación deportiva implica relacionar variables tales como el rendimiento físico inicial, la deserción deportiva y el vínculo familiar para la toma de acciones estratégicas acertadas. Por ello, el objetivo de la investigación es elaborar un grupo de acciones que permita estimular la iniciación y permanencia de las niñas identificadas como talentos, para la práctica del Atletismo infantil en La Federación Deportiva de Loja. La investigación se enmarca en las características de la descripción, la transversalidad y la correlación. Se estudian a 30 atletas, sexo femenino (8-11 años), y a 56 padres de ellas mediante la técnica de encuesta, aplicando además los test de Estatura, Rapidez (30m), Salto Largo, Abdominales, Planchas y Resistencia (400m). A partir de la descripción de las potencialidades individuales de las atletas, y de los familiares, se diseñaron 13 acciones estratégicas para potenciar la permanencia de las atletas en los entrenamientos, permitiendo mantener la comunicación entre la familia, las niñas, el profesorado y los entrenadores.

Palabras Claves: Atletismo. Impulsión de la Bala, Análisis Biomecánico.

\section{Introducción.}

El deporte constituye una eficaz herramienta de comunicación por su enorme capacidad de convocatoria regional, nacional e internacional (Sanjurjo, 2012; Camino \& Ayala, 2012), las actividades deportivas son un poderoso instrumento de comunicación y una magnífica oportunidad para transmitir mensajes importantes.

Los deportes se relacionan estrechamente con el potencial humano (Chelladurai \& Kerwin, 2017; Knudson, 2013), el esfuerzo y la consecución del éxito, y tienen la facultad de emocionar y levantar el ánimo (McGannon \& Smith, 2015).

El Atletismo, es un deporte que comprende un conjunto de disciplinas agrupadas en carreras, saltos, lanzamientos, pruebas combinadas y marcha (Seners, 2001; Granell \& Lazcorreta, 
2004). Es el arte de superar el rendimiento de los adversarios en velocidad, en resistencia y en distancia al lanzar o saltar.

Por la gran variedad de acciones que configuran sus diferentes modalidades, en cada una de estas pruebas se demuestran distintas habilidades físicas y técnicas que permiten el desarrollo integral de los(as) practicantes (Gamble, 2013; Beattie, Kenny, Lyons, \& Carson, 2014; Del Coso, y otros, 2014).

La iniciación deportiva en la etapa infantil permite realizar la preparación del niño(a) durante un largo período de tiempo (Martin \& Nicolaus, Metodología general del entrenamiento infantil y juvenil, 2004; Núñez, 2000); por lo que resulta importante el trabajo referido a las actividades deportivas desde edades tempranas.

El trabajo multilateral que se realiza en las áreas deportivas, garantiza el futuro del alto rendimiento en Ecuador (Martin, Entrenamiento multilateral y especialización precoz, 1993; Bompa, 2005). La masificación deportiva es importante dado que es de donde surgen los talentos. Es criterio de los especialistas del Atletismo que la iniciación temprana constituye el proceso de enseñanza preliminar de los elementos básicos de este deporte (Ernesto Avella \& Medellín, 2013).

En la actualidad, resulta necesario consolidar la práctica masiva del atletismo entre niños(as), para lograr una reserva atlética adecuada desde las edades tempranas en el Atletismo femenino, para la cual la República del Ecuador, al poseer varios resultados en algunas modalidades del atletismo, es imprescindible establecer las estrategias pertinentes a todos los niveles para potenciar la masificación deportivas en general, pero especialmente establecer los indicadores necesarios para la selección científica del deportista de atletismo según las modalidades o disciplinas disponibles.

Como parte de la consulta de fuentes primarias de investigación, algunas obras en el Ecuador señalan la importancia del proceso de selección de talentos deportivos en diferentes disciplinas del atletismo (Morales \& Priscila, 2016), aunque la literatura en lo fundamental trabaja aspectos importante de la preparación como la atención a la preparación física (Pico Medina \& Brazales Cervantes, 2014) y la planificación de las cargas del entrenamiento 
adaptada al entorno especifico ecuatoriano (Espinoza Loja, 2009), así como otros componentes como la preparación psicológica (Marín \& Eduardo, 2013), entre otros.

El énfasis del presente trabajo de titulación, se relaciona con la potenciación de la selección deportiva en el atletismo atendiendo a componentes de la selección deportiva, destacando las características necesarias atendiendo a los componentes motivacionales del alumnado para disminuir la deserción deportiva, factor éste de suma importancia en la teoría y metodología del deportista (López-Walle, Tristán,, Cantú-Berrueto, Zamarripa, \& Cocca, 2013; Chávez $\&$ Calero, 2015), que para el caso ecuatoriano inciden varios aspectos en ello, tal y como se platea en algunas obras (Chávez Hernández, 2015), pero que aún no están estudiadas a profundidad, dado la escases de trabajos consultados para diferentes deportes en general, y mucho menos del atletismo como deporte específico.

Dado lo anterior, el objetico del paper es elaborar un grupo de acciones que permita estimular la iniciación y permanencia de las niñas identificadas como talentos, para la práctica del Atletismo infantil en La Federación Deportiva de Loja.

\section{Métodos.}

La investigación se enmarca en las características de la descripción, la transversalidad y la correlación. La investigación estudiará una muestra representativa de la población de atletas del sexo femenino aspirantes a deportistas de atletismo en la etapa de iniciación (8-11 años de edad) de la Federación Deportiva de Loja, la cual fue delimitada por medio de un muestreo aleatorio simple, estableciendo la muestra en 30 sujetos, de los cuales son descritos sus datos básicos a partir del capítulo 3 como parte del análisis de los resultados.

Por otra parte, como parte del proceso de investigación, y con el propósito de conocer aspectos de interés sobre la influencia de los padres en el proceso de dirección del entrenamiento deportivo del atletismo, se encuestó a los padres de la muestra seleccionada (56 padres), siendo el valor mencionado la población existente de familiares directos con vida. En la aplicación del método de encuesta, se incluyó a 10 profesores de educación física que participan en los procesos de selección deportiva en la provincia donde se realiza la 
investigación, delimitando algunos aspectos de interés relacionados con el proceso investigado. La información de interés será recolectada por medio de especialistas con al menos 10 años de experiencia práctica en la profesión, con titulación de pregrado universitario (licenciado en Cultura Física o Actividad Física, Deportes y Recreación). Los especialistas ascienden a 5 sujetos. En algunas pruebas de valoración del rendimiento deportivo los cinco especialistas trabajarán en conjunto (rapidez y resistencia), en otras solo se emplearán dos especialistas (Estatura, Salto, abdominales, lagartijas).

Para el cálculo que determina la representatividad de la muestra, se empleó la fórmula del muestreo irrestricto aleatorio, delimitando como tamaño de muestra idónea a 30 sujetos del sexo femenino aspirantes a deportistas de alto rendimiento de diferentes modalidades de atletismo. El Microsoft Excel 2016 será el procesador estadístico de excelencia, diseñándose previamente unas plantillas para el registro y procesamiento de la información de interés.

La metodología de los test pedagógicos a emplear se evidencia a continuación:

1) Estatura: La metodología empleada para medir la estatura de las niñas es la siguiente: La niña descalza, parada derecha con pies unidos en los talones, puntas de los pies ligeramente abiertas, brazos abajo y cabeza mirando hacia delante, de manera que la línea entre el orificio auricular y el ángulo del ojo describa una paralela con relación al piso, la espalda lo más próxima posible a la pared donde se encuentra situada la escala de medidas elaborada en la pared, se tomó una regla, se colocó sobre la superficie más alta de la cabeza y se obtuvo la talla en centímetro.

2) Rapidez (30 metros planos): La niña se ubica con la pierna delantera sobre la línea de arrancada (la arrancada desde la posición de parada) a la orden de arrancada, correr sin disminuir la velocidad hasta pasar rápido por la línea de meta, tomándose el tiempo en segundos con la mayor precisión posible al pasar la línea de meta y correrán de una en una.

3) Salto largo sin carrera de impulso: En una superficie plana de tres metros de largo como mínimo y uno de ancho, antirresbalable marcada en centímetros en el piso con una tiza. La niña se sitúa de tal forma que la punta de los pies quede detrás de la línea de despegue y con las piernas ligeramente separadas. En el momento en que se encuentre preparada, saltará hacia delante buscando la máxima distancia; para ello 
realizará un balanceo de brazos hacia atrás y simultáneamente con el movimiento de los mismos hacia delante, despegará con ambas piernas al mismo tiempo, se mide desde la última huella, la distancia se mide en centímetros y se tomará el mejor de los dos intentos.

4) Abdominales: En una superficie lisa, la niña sentada se acuesta con la espalda sobre la superficie, con los pies separados a una distancia de $30 \mathrm{~cm}$., las piernas flexionadas en las rodillas en un ángulo recto. Los brazos irán cruzados al pecho. Otra niña que hace de la pareja se apoya frente a las piernas de la que vas a realizar prueba y se las sostiene por los tobillos de forma tal que mantenga siempre los talones sobre la superficie. Desde la posición de acostada irá a la de sentada sin llegar a la línea media vertical. Sin parar regresará a la posición inicial de forma tal que la espalda toque superficie para inmediatamente sentarse de nuevo y repetir la acción. Se cuenta la cantidad de movimientos completos.

5) Planchas o Lagartijas: En una superficie plana preferiblemente de cemento. La niñas se sitúa con la parte delantera del cuerpo sobre la superficie plana sin irregularidades, brazos flexionados, manos apoyadas al nivel de las axilas, los dedos hacia el frente, la cabeza en línea recta con el tronco, vista paralela con la superficie; con apoyo de las rodillas. Se cuidará que en cada extensión de brazos el cuerpo se mantenga en forma recta; se controlarán todas las repeticiones hasta que se altere la correcta ejecución técnica del movimiento.

6) Resistencia 400 metros planos: En una pista sintética ó de tierra con la medida homologada (400 M) se corre 400 metros. Las niñas situadas detrás de la línea de arrancada, a la orden de salida se toma el tiempo en minutos con un cronómetro manual, cuando pase por la línea de meta se toma el tiempo. En esta prueba pueden correr hasta 8 niñas al salir de la línea de arrancada se dirigen todas hacia el carril N0.1 Al llegar a la meta se le entrega un cartón con el número por el orden de llegada para luego registrar el tiempo plasmado en el cronómetro en el registro. 


\section{Resultados y discusión.}

Los resultados de la encuesta aplicada a las atletas se valoran a continuación:

Tabla 1: Desglose de la edad por rangos etario

\begin{tabular}{lrrrrc}
\hline \multicolumn{1}{c}{ No } & 8 años & 9 años & 10 años & 11 años & Total \\
\hline FA & 5 & 8 & 10 & 7 & $\mathbf{3 0}$ \\
FP & $16,67 \%$ & $26,67 \%$ & $33,33 \%$ & $23,33 \%$ & \\
\hline
\end{tabular}

Según se establece en la tabla 1, los rangos estarios de la investigación se establecieron entre los 8-11 años de edad, siendo el 16,67\% los comprendidos en los 8 años en términos e Frecuencia Porcentual (FP) con cinco sujetos como Frecuencia Absoluta. La cantidad de estudiados comprendidos en los 9 años suman 8 sujetos (16,67\%), en los comprendidos en los 10 años se estudiaron a 10 sujetos (33,33\%), y los sujetos comprendidos en los 11 años se estudiaron a 7 posibles atletas $(23,33 \%)$.

Tabla 2: Sobre los gustos por la actividad física practicada.

\begin{tabular}{lrrrrrr}
\hline No & $\begin{array}{c}\text { Se } \\
\text { divierte }\end{array}$ & $\begin{array}{c}\text { Desea tener } \\
\text { nuevas amistades }\end{array}$ & $\begin{array}{c}\text { Desea ser } \\
\text { deportista }\end{array}$ & $\begin{array}{c}\text { Desea ser } \\
\text { campeona en } \\
\text { torneos }\end{array}$ & $\begin{array}{c}\text { Desea mejorar } \\
\text { su salud }\end{array}$ & Total \\
\hline FA & 9 & 7 & 14 & 5 & 1 & $\mathbf{3 0}$ \\
\hline PP & $30,00 \%$ & $23,33 \%$ & $46,67 \%$ & $16,67 \%$ & $3,33 \%$ & \\
\hline
\end{tabular}

En términos de porcentajes, en nueve sujetos estudiados (30\%) existe la diversión en al instante de practicar actividad física especializada, mientras en que siete sujetos se denotó el deseo de tener nuevas amistades (23,33\%), un aspecto que la actividad física suele favorecer. Para el caso del deseo de ser deportista, solamente 14 sujetos respondieron que sí $(46,67 \%)$, un porcentaje que a consideración de los autores es pequeño, por lo cual es necesario establecer a futuro estrategias de motivación. Para el caso del deseo de ser campeona en algún torneo, solamente se expresaron positivamente 5 sujetos $(16,67 \%)$, un aspecto a entender como positivo, dado que el campeonismo no es una motivación esencian en las edades tempranas estudiadas, aunque podría ser una fuerte motivación en la muestra pequeña señalada. En términos de obtención de salud, solamente una deportista señalo de idea 
$(3,33 \%)$, aspecto que por lógica no debía existir en dichas edades, dado que los niños en edades tempranas no suelen pensar en dichos problemas, aunque la niña que boto por dicha opción tiene un sobrepeso, aunque dicha condición somatotipica suele ser importante para varias modalidades del atletismo, como lo es el lanzamiento de la bala.

Tabla 3: Lo que más gusta de la práctica del atletismo.

\begin{tabular}{cccccccc}
\hline No & $\begin{array}{c}\text { Correr } \\
\text { Velocidad }\end{array}$ & $\begin{array}{c}\text { Correr } \\
\text { Vallas }\end{array}$ & $\begin{array}{c}\text { Correr } \\
\text { Resistencia }\end{array}$ & Jugar & $\begin{array}{c}\text { Salto } \\
\text { Alto }\end{array}$ & $\begin{array}{c}\text { Salto } \\
\text { Largo }\end{array}$ & Total \\
\hline FA & 9 & 5 & 5 & 10 & 3 & 1 & $\mathbf{3 0}$ \\
PP & $30,00 \%$ & $16,67 \%$ & $16,67 \%$ & $33,33 \%$ & $10,00 \%$ & $3,33 \%$ & \\
\hline
\end{tabular}

Los porcentajes presentes en la tabla 3, evidencian cuáles son los gustos que poseen la atletas encuestadas por la práctica de atletismo, evidenciando que en la modalidad de CorrerVelocidad 9 sujetos estaban interesadas (30\%), en la modalidad de Correr-Vallas, se interesaron 5 atletas (16,67\%), en la modalidad de Correr-Resistencia se motivaron 5 sujetos $(16,67 \%)$, para el caso del juego el porcentaje fue el mayor de todos con 10 atletas motivadas $(33,33 \%)$, infiriendo que el método de juego es fundamental en la edad. Por otra parte, para el caso de la modalidad de Salto Alto, se interesaron solamente 3 sujetos (10\%), y para el caso de la modalidad de Salto Largo se interesaron solamente una atleta $(3,33 \%)$, infiriendo que para las modalidades de salto las motivaciones suelen ser menores en el rango etario estudiado.

Tabla 4: Lo que menos gusta de la práctica de atletismo.

\begin{tabular}{ccccccccc}
\hline No & $\begin{array}{c}\text { Correr } \\
\text { Velocidad }\end{array}$ & $\begin{array}{c}\text { Correr } \\
\text { Vallas }\end{array}$ & $\begin{array}{c}\text { Correr } \\
\text { Resistencia }\end{array}$ & Jugar & $\begin{array}{c}\text { Salto } \\
\text { Alto }\end{array}$ & $\begin{array}{c}\text { Salto } \\
\text { Largo }\end{array}$ & Abd & Total \\
\hline FA & 3 & 7 & 23 & 0 & 3 & 2 & 5 & $\mathbf{3 0}$ \\
PP & $10,00 \%$ & $23,33 \%$ & $76,67 \%$ & $0,00 \%$ & $10,00 \%$ & $6,67 \%$ & $16,67 \%$ & \\
\hline
\end{tabular}

Para el caso de las actividades que menor gustaron a la muestra estudiada (Tabla 4), los porcentajes evidencia que el juego no posee problemas $(0 \%)$, o sea a todos de alguna manera les gusta el juego, reforzando la idea expresada como parte del análisis de la tabla 3 , de que el métodos de juego debe priorizarse en el diseño de la preparación deportiva.

El resto de los porcentajes se expresaron como factor de desmotivación en la práctica de la modalidad deportiva la especialidad de Correr-Velocidad, con 3 atletas que para nada les gusta dicha modalidad deportiva del atletismo (10\%), para la modalidad de Correr-Vallas el 
porcentajes se estableció en 23,33\% ( 7 sujetos), para el caso de la modalidad CorrerResistencia el porcentaje de desmotivación es el más elevado (76,67\%) existiendo en 23 sujetos de un total de 30 estudiados. Por otra parte, la desmotivación por el Salto Alto se estableció en el 10\% (3 sujetos), y la desmotivación por el Salto Largo se estableció en el $6,67 \%$ de la muestra estudiada ( 2 sujetos).

Para el caso de los abdominales (Abd), una variable incluida a última hora en la investigación, se estableció como factor de desmotivación en el 16,67\% de la muestra (5 sujetos).

Tabla 5: Tener temor a perder competiciones

\begin{tabular}{lccccc}
\hline No & SI & NO & $\begin{array}{c}\text { Algunas } \\
\text { Veces }\end{array}$ & Siempre & Total \\
\hline FA & 0 & 24 & 4 & 2 & $\mathbf{3 0}$ \\
PP & $0,00 \%$ & $80,00 \%$ & $13,33 \%$ & $6,67 \%$ & \\
\hline
\end{tabular}

En la respuesta sobre el temor a perder competiciones (Tabla 5), el 100 por ciento en sentido general evidenció la inexistencia de dicho indicador, por lo cual las 30 chicas determinaron en un cero por ciento $(0 \%)$ la respuesta de Sí, infiriendo para la primera vuelta de la encuesta (antes de la competencia) la inexistencia de miedo. El 80\% de la muestra estudiada evidenció con seguridad que No tiene miedo a las competiciones ( 24 sujetos) en la primera vuelta de la encuesta (antes de la competencia). Por otra parte, luego de competir se aplicó una segunda vuelta en la aplicación de la encueta, determinándose que el 13,33\% confeso que tenía algunas veces miedo (4 sujetos), y que el 6,67\% (2 sujetos), estuvo con miedo, aspecto que como acciones como parte del proceso de dirección del entrenamiento hay que tener presente para contrarrestar y evitar los problemas de rendimiento asociados.

Tabla 6: Cuándo pierde en las competencias piensa:

\begin{tabular}{lcccc}
\hline No & $\begin{array}{c}\text { No practicar } \\
\text { más }\end{array}$ & $\begin{array}{c}\text { Practicar más } \\
\text { fuerte }\end{array}$ & Otros & Total \\
\hline FA & 4 & 21 & 5 & $\mathbf{3 0}$ \\
PP & $13,33 \%$ & $70,00 \%$ & $16,67 \%$ & \\
\hline
\end{tabular}

Los pensamientos o acciones del comportamiento al finalizar una competencia al momento de perder o valorar la posibilidad de perder se evidencia en la tabla 6. Los resultados 
evidenciaron que el 13,33\% (4 sujetos) no deseaban practicar más atletismo, mientras que los 21 casos $(70 \%)$ sentían el deseo de practicar aún más para mejorar los resultados competitivos. Por otra parte, en 5 sujetos $(16,67 \%)$ se evidenciaron otras causas aisladas, como la necesidad de obtener reconocimiento familiar, del entrenador y de amigos, indiferencia entre otras causas.

Tabla 7: De las competiciones y los test pedagógicos aplicados en el atletismo qué es lo que menos le gusta.

\begin{tabular}{cccccccc}
\hline No & $\begin{array}{c}\text { Velocidad } \\
\mathbf{4 0 m}\end{array}$ & $\begin{array}{c}\text { Resistencia } \\
\mathbf{1 0 0 0 m}\end{array}$ & $\begin{array}{c}\text { Salto } \\
\text { Alto Tijera }\end{array}$ & $\begin{array}{c}\text { Salto } \\
\text { Largo }\end{array}$ & $\begin{array}{c}\text { Lanzamiento } \\
\text { Balón }\end{array}$ & $\begin{array}{c}\text { Correr } \\
\text { Vallas }\end{array}$ & Total \\
\hline FA & 3 & 24 & 3 & 0 & 1 & 2 & $\mathbf{3 0}$ \\
PP & $10,00 \%$ & $80,00 \%$ & $10,00 \%$ & $0,00 \%$ & $3,33 \%$ & $6,67 \%$ & \\
\hline
\end{tabular}

De las competiciones y los test pedagógicos aplicados en el atletismo que menos les gustan a la muestra estudiada el mayor porcentaje se determinó en el test de Resistencia de los $1000 \mathrm{~m}$, con un porcentaje del 80\% (24 sujetos), dato que evidencia una aversión por la capacidad física de resistencia, capacidad vital en muchas modalidades deportivas del atletismo, pero que deben potenciarse con diversas estrategias en las edades tempranas. Seguidamente, para el caso de los test de Velocidad (40m) y el test de Salto Alto en Tijeras, los porcentajes mostraron poco gusto en la muestra estudiada en un porcentaje para ambos del 10\% (3 sujetos). Para el salto Largo no se evidenció desmotivación para su práctica (0\%), para el caso del Lanzamiento del Balón solamente el 3,33\% (1 sujeto) de la muestra estudiada mostró poco gusto en su ejecución, y para el test o modalidad competitiva de Correr con Vallas, solamente un porcentaje del 6,67\% (2 sujetos) determinaron poco gusto por su ejecución.

Los datos reflejados en las tablas anteriores, como parte de la encuesta aplicada a la muestra de niñas deportistas estudiadas, permite aclarar algunas ideas sobre la selección deportiva en términos de individualidad, y sobre todo como aspecto a tener presente para el diseño de acciones de trabajo que permitan cumplimentar la hipótesis y objetivo trazado en la presente investigación.

Los resultados de la encuesta aplicada a las familias de las atletas se valoran a continuación: 
Tabla 8: Nivel de escolaridad de los padres encuestados

\begin{tabular}{lcccc}
\hline No & Universitario & $\begin{array}{c}\text { Medio } \\
\text { Superior }\end{array}$ & Otros & Total \\
\hline FA & 13 & 27 & 16 & $\mathbf{5 6}$ \\
PP & $23,21 \%$ & $48,21 \%$ & $28,57 \%$ & \\
\hline
\end{tabular}

Sobre el nivel de escolaridad de los padres de las atletas estudiadas, la tabla 7 evidencia que el nivel universitario de encuentra en un $23,21 \%$ (13 sujetos) por ciento del total encuestado (56 padres), denotando el índice más bajo del nivel de escolaridad. Seguidamente, los niveles más altos por orden se establecen en el nivel Medio Superior con un 48,21\% (27 sujetos), y en el nivel "Otros" con un porcentaje de 28,57\% (16 sujetos). Lo anterior indica que el nivel de escolaridad suele ser bajo con respecto a otros países desarrollados e incluso del entorno geográfico cercano. El aspecto valorado con anterioridad es vital para el diseño de las acciones pertinentes que estén acordes a las necesidades a cumplimentar |por cada clase social.

Tabla 9: ¿Trabajan?

\begin{tabular}{cccc}
\hline No & SI & NO & Total \\
\hline FA & 37 & 19 & $\mathbf{5 6}$ \\
PP & $66,07 \%$ & $33,93 \%$ & \\
\hline
\end{tabular}

La parte de la encuesta encargada de valorar los índices laborales de la familia se valoran en la tabla 8 . Se evidencia que el 66,07\% (37 sujetos) trabajan en diversos centros laborales, mientras que el 33,93\% (19 sujetos) no trabajan, o son amas de casas.

Tabla 10: Les resulta agradable que la deportista de su familia práctica atletismo

\begin{tabular}{cccc}
\hline No & SI & NO & Total \\
\hline FA & 56 & 0 & $\mathbf{5 6}$ \\
PP & $100,00 \%$ & $0,00 \%$ & \\
\hline
\end{tabular}

Sobre si a la familia le resultaba agradable que la deportista practicará atletismo, la tabla 9 evidenció que el $100 \%$ de la muestra tenía interés por que su familiar practicará el atletismo, indistintamente de modalidad deportiva seleccionada. 
Tabla 11: Lo que más le agradaría que practicará su niña.

\begin{tabular}{cccccccc}
\hline No & Atletismo & Baile & Computación & idiomas & $\begin{array}{c}\text { Artes } \\
\text { Marciales }\end{array}$ & Modelaje & Total \\
\hline FA & 41 & 9 & 6 & 4 & 0 & 5 & $\mathbf{5 6}$ \\
PP & $73,21 \%$ & $16,07 \%$ & $10,71 \%$ & $7,14 \%$ & $0,00 \%$ & $8,93 \%$ & \\
\hline
\end{tabular}

Qué es lo que más le agradaría al familiar que practicará la atleta se evidencia en la tabla 10. El mayor porcentaje se evidenció en el propio atletismo con un 73,21\% (41 sujetos) del total encuestado, aspecto que evidencia que la mayoría de los familiares están satisfechos con la práctica del deporte por parte de su familiar. Por otra parte, en términos de porcentajes los mayores valores se establecieron en el Baile como modalidad de recreación activa en esencial (16,07\%: 6 sujetos), seguido por la computación (recreación pasiva) con un 10,71\% (6 sujetos), el Modelaje con un 8,93\% (5 sujetos), y finalizando por los Idiomas en un 7,14\% (4 sujetos) del total de la muestra de familiares estudiados.

Tabla 12: Han practicado algún deporte?

\begin{tabular}{lccc}
\hline \multicolumn{1}{c}{ No } & SI & NO & Total \\
\hline FA & 7 & 49 & $\mathbf{5 6}$ \\
PP & $12,50 \%$ & $87,50 \%$ & \\
\hline
\end{tabular}

El estudio sobre sí algún familiar de las atletas han practicado algún deporte durante su vida activa, la tabla 11 evidenció que un pequeño porcentaje determinó que Sí (12,5\%: 7 sujetos), siendo la mayoría de los familiares ajenos en términos prácticos a la práctica deportiva, dado que un porcentaje abrumador fue determinado en la respuesta de NO, con un 87,5\% (49 sujetos) del total de los familiares estudiados. Si bien no se evidencia en el presente estudio que los miembros familiares de las atletas en términos generales no posee una experiencia sistemática en la práctica deportiva, existe el consenso familiar de la importancia que en términos generales posee la actividad física, especialmente la práctica deportiva, tanto para aspectos relacionados con la salud, la educación física e intelectual, el uso correcto del tiempo libre, y el vínculo o relación del apoyo familiar en los gustos y preferencias de los integrantes más jóvenes del núcleo familiar. 
Tabla 13: Cree que sea necesario rescatar la carga académica concerniente a la práctica deportiva en las escuelas ecuatorianas.

\begin{tabular}{lccc}
\hline \multicolumn{1}{c}{ No } & SI & NO & Total \\
\hline FA & 56 & 0 & $\mathbf{5 6}$ \\
PP & $100,00 \%$ & $0,00 \%$ & \\
\hline
\end{tabular}

Sobre la necesidad que la familia valora en términos de rescatar la carga académica concerniente a la práctica deportiva en las escuelas ecuatorianas, existió un consejo total de los familiares encuestados por el Sí (100\%), tal y como se evidencia en la tabla 12.

Tabla 14: Cómo valora el deporte?

\begin{tabular}{lccccc}
\hline No & $\begin{array}{c}\text { Útil } \\
\text { Salud }\end{array}$ & $\begin{array}{c}\text { Útil-Iniciación } \\
\text { Deportiva }\end{array}$ & $\begin{array}{c}\text { Útil-Formación } \\
\text { Integral }\end{array}$ & $\begin{array}{c}\text { Sin } \\
\text { Importancia }\end{array}$ & Total \\
\hline FA & 11 & 24 & 21 & 0 & $\mathbf{5 6}$ \\
PP & $19,64 \%$ & $42,86 \%$ & $37,50 \%$ & $0,00 \%$ & \\
\hline
\end{tabular}

La familia valora la práctica deportiva teniendo presente algunos indicadores demostrados en la literatura internacional (Tabla 13). Para el caso de la valoración de la relación de la práctica deportiva con la salud (Útil Salud), se evidenció que el 19,64\% (11 sujetos) consideraba la realidad y necesidad de vincular a su familiar atleta en el deporte dado sus ventajas en brindar salud corporal y psicológica al deportista. Un 42,86\% (2 sujetos) cree que la práctica temprana del deporte es útil para la iniciación deportiva y el alcance de altos rendimientos deportivos, mientras que un 37,5\% (21 sujetos) de los familiares valora la importancia de la práctica deportiva en la formación integral de la persona. Por otra parte, ningún encuesto valoró la práctica deportiva como algo sin importancia ( $0 \%)$. El análisis de los datos antes mencionado, deduce de que a pesar de que un mayor porcentaje de los familiares no tenga educación superior, posee los conocimientos necesarios para considerar las ventajas implícitas de la práctica sistemáticas de actividad física y deportes, aspecto a potenciar por parte de los entrenadores, sobre todo para aquellos que tengan dudas al respecto. 
Tabla 15: Disposición para apoyar a las chicas en la práctica del atletismo.

\begin{tabular}{lccc}
\hline \multicolumn{1}{c}{ No } & SI & NO & Total \\
\hline FA & 56 & 0 & $\mathbf{5 6}$ \\
PP & $100,00 \%$ & $0,00 \%$ & \\
\hline
\end{tabular}

Sobre la disposición de los familiares para apoyar a las chicas en la práctica del atletismo, se coincidió en un 100\% que Sí (56 Sujetos). Por lo cual, se evidencia un bajo freno familiar en la potenciación de la práctica deportiva en general, y en la práctica del atletismo en particular.Los resultados de las entrevistas informales a los familiares y profesores de las atletas se listan a continuación:

1) Los profesores de educación física de las escuelas dieron su criterio de las niñas con potencialidades para el Atletismo, mostrando las niñas que se destacan en la Educación Física; proporcionando agilidad en cuanto a conocer de la existencia de las potencialidades de las niñas .

2) Las madres, fundamentalmente las que trabajan, plantearon que le resulta muy difícil poder llevar las niñas al área deportiva, teniendo temor de mandarlas solas por diversos motivos. Algunos familiares refieren afectaciones psicológicas de la menor por separación de los padres y plantearon que el deporte la ha ayudado.

3) En relación con la baja incorporación, los familiares manifestaron que existen factores que conspiran contra la práctica del deporte tales como: El horario docente, el volumen elevado de tareas y trabajos prácticos, no contar con una persona que pueda llevar la niña al deporte, entre otros.

4) Sobre las entrevista informales a profesores vinculados al proceso docente-educativo general de las chicas, al solicitarle su opinión en relación con su valoración y apoyo de las actividades Deportivas Recreativas en la escuela, los clubes deportivos, y el barrio, refirieron que resultaba interesante y que estarían en la mejor disposición de formar parte del equipo de trabajo, dado que en sus planes de trabajo ellos deben realizar actividades deportivas, con énfasis en fechas históricas, que estaban en la mejor disposición de apoyar para lograr la realización de las mismas y que sí se trataba de la búsqueda de talentos para el atletismo lo valoran como interesante, que lo perciben como una buena opción que a nivel comunitario que se oferten Actividades Deportivas Recreativas y Culturales para todas las edades, de 
ISSN: 2602-8085

esta manera al realizarse en la propia comunidad de forma organizada, todos tienen oportunidades de participar incluyendo al núcleo familiar, al menos como espectador.

Las pruebas de valoración del rendimiento deportivo para las atletas que determinan el rendimiento inicial se listan en las tablas 15 y 16.

Tabla 16: Grupo 1 de pruebas de valoración del rendimiento deportivo

\begin{tabular}{|c|c|c|c|c|c|c|c|c|c|c|}
\hline No & Edad & Estatura & $\mathbf{N}$ & $\mathrm{C}$ & $\begin{array}{c}\text { Rapidez } \\
\text { 30m } \\
\end{array}$ & $\mathbf{N}$ & $\mathrm{C}$ & $\begin{array}{c}\text { Salto } \\
\text { Largo } \\
\end{array}$ & $\mathbf{N}$ & $\mathrm{C}$ \\
\hline 1 & 8 & 135 & 138 & $\mathrm{NO}$ & 6,9 & 6,8 & $\mathrm{NO}$ & 120 & 125 & $\mathrm{NO}$ \\
\hline 2 & 8 & 133 & 138 & NO & 7,3 & 6,8 & NO & 121 & 125 & NO \\
\hline 3 & 8 & 134 & 138 & $\mathrm{NO}$ & 7 & 6,8 & $\mathrm{NO}$ & 123 & 125 & $\mathrm{NO}$ \\
\hline 4 & 8 & 140 & 138 & SI & 6,6 & 6,8 & SI & 120 & 125 & $\mathrm{NO}$ \\
\hline 5 & 8 & 137 & 138 & NO & 7,1 & 6,8 & $\mathrm{NO}$ & 118 & 125 & $\mathrm{NO}$ \\
\hline 6 & 9 & 140 & 145 & $\mathrm{NO}$ & 6,9 & 6,5 & NO & 133 & 137 & $\mathrm{NO}$ \\
\hline 7 & 9 & 142 & 145 & $\mathrm{NO}$ & 6,5 & 6,5 & SI & 138 & 137 & SI \\
\hline 8 & 9 & 143 & 145 & NO & 6,7 & 6,5 & $\mathrm{NO}$ & 132 & 137 & NO \\
\hline 9 & 9 & 140 & 145 & $\mathrm{NO}$ & 6,9 & 6,5 & $\mathrm{NO}$ & 137 & 137 & $\mathrm{NO}$ \\
\hline 10 & 9 & 145 & 145 & SI & 6,6 & 6,5 & NO & 140 & 137 & SI \\
\hline 11 & 9 & 135 & 145 & NO & 6,8 & 6,5 & $\mathrm{NO}$ & 136 & 137 & $\mathrm{NO}$ \\
\hline 12 & 9 & 134 & 145 & NO & 7 & 6,5 & $\mathrm{NO}$ & 141 & 137 & SI \\
\hline 13 & 9 & 136 & 145 & NO & 6,3 & 6,5 & SI & 132 & 137 & NO \\
\hline 14 & 10 & 139 & 151,5 & NO & 6,6 & 6,4 & $\mathrm{NO}$ & 130 & 138 & $\mathrm{NO}$ \\
\hline 15 & 10 & 136 & 151,5 & NO & 6,4 & 6,4 & SI & 137 & 138 & NO \\
\hline 16 & 10 & 141 & 151,5 & NO & 6,3 & 6,4 & SI & 135 & 138 & NO \\
\hline 17 & 10 & 140 & 151,5 & $\mathrm{NO}$ & 6,7 & 6,4 & $\mathrm{NO}$ & 132 & 138 & $\mathrm{NO}$ \\
\hline 18 & 10 & 142 & 151,5 & NO & 6,5 & 6,4 & NO & 137 & 138 & NO \\
\hline 19 & 10 & 138 & 151,5 & NO & 6,2 & 6,4 & SI & 139 & 138 & SI \\
\hline 20 & 10 & 137 & 151,5 & $\mathrm{NO}$ & 6 & 6,4 & SI & 140 & 138 & SI \\
\hline 21 & 10 & 138 & 151,5 & NO & 6,3 & 6,4 & SI & 131 & 138 & NO \\
\hline 22 & 10 & 142 & 151,5 & NO & 6,9 & 6,4 & NO & 136 & 138 & $\mathrm{NO}$ \\
\hline 23 & 10 & 145 & 151,5 & NO & 7 & 6,4 & NO & 132 & 138 & NO \\
\hline 24 & 11 & 145 & 157 & $\mathrm{NO}$ & 6,6 & 6,3 & $\mathrm{NO}$ & 137 & 145 & $\mathrm{NO}$ \\
\hline 25 & 11 & 141 & 157 & $\mathrm{NO}$ & 6,1 & 6,3 & SI & 141 & 145 & $\mathrm{NO}$ \\
\hline 26 & 11 & 140 & 157 & NO & 6,4 & 6,3 & NO & 140 & 145 & NO \\
\hline 27 & 11 & 143 & 157 & NO & 6,4 & 6,3 & $\mathrm{NO}$ & 151 & 145 & SI \\
\hline 28 & 11 & 148 & 157 & NO & 6,2 & 6,3 & SI & 143 & 145 & NO \\
\hline 29 & 11 & 138 & 157 & NO & 6 & 6,3 & SI & 141 & 145 & $\mathrm{NO}$ \\
\hline 30 & 11 & 157 & 157 & SI & 6,1 & 6,3 & SI & 140 & 145 & NO \\
\hline M & 9,63 & 140,13 & & $10,00 \%$ & 6,58 & & $40,00 \%$ & 134,43 & & $20,00 \%$ \\
\hline
\end{tabular}


De los 30 sujetos estudiados, la tabla 15 especifica los resultados de las pruebas de valoración del rendimiento concernientes a la Estatura, la Rapidez y el Salto Largo sin Carrera de Impulso en conjunto con las normas o baremos que evalúan una estatura adecuada a los requerimientos internacionales $(\mathrm{N})$, así como el cumplimiento o no de la misma $(\mathrm{C})$. Las medias o promedios obtenidos $(\mathrm{M})$ establecieron para el caso de la estatura unos $1.40,13 \mathrm{~cm}$, para la media de edad entre los 8-11 años de 9,63, cumplimentando la norma solamente el $10 \%$ de la muestra (3 sujetos). Por otra parte, la media de la muestra estudiada para el caso del Test de rapidez (30m) se estableció en 6:58sg, cumplimentando la norma solamente el $40 \%$ de la muestra (12 sujetos). Para el caso de la prueba de Salto Largo sin Carrera de Impulso la media de la muestra estudiada se estableció en $134,43 \mathrm{~cm}$, cumpliendo las normativas exigidas para cada edad solamente el $20 \%$ de la muestra (6 sujetos).

Para el caso de las tres pruebas mencionadas con anterioridad los baremos establecidos fueron:

a) El percentil/ establecido para cada rango de edad para la estatura en centímetros es el siguiente:

1) 8 años $138 \mathrm{~cm}$

2) 9 años $145 \mathrm{~cm}$

3) 10 años $151.5 \mathrm{~cm}$

4) 11 años $157 \mathrm{~cm}$

b) El percentil establecido para cada rango de edad que delimita el rendimiento de la capacidad física rapidez $(30 \mathrm{~m})$ es el siguiente:

1) 8 años $6.8 \mathrm{sg}$

2) 9 años $6.5 \mathrm{sg}$

3) 10 años $6.4 \mathrm{sg}$

4) 11 años $6.3 \mathrm{sg}$

c) Las normativas en centímetros establecidas para el primer nivel por rango de edad para la prueba de Salto Largo sin Carrera de Impulso son las siguientes:

1) 8 años: $125 \mathrm{~cm}$

2) 9 años: $137 \mathrm{~cm}$

3) 10 años: $138 \mathrm{~cm}$ 
4) 11 años: $145 \mathrm{~cm}$

El segundo grupo de pruebas de valoración del rendimiento deportivo aplicadas se valora en la tabla 16.

Tabla 17: Grupo 2 de pruebas de valoración del rendimiento deportivo

\begin{tabular}{|c|c|c|c|c|c|c|c|c|c|c|}
\hline No & Edad & Planchas & $\mathbf{N}$ & $\mathbf{C}$ & Abd & $\mathbf{N}$ & $\bar{C}$ & $\begin{array}{l}\text { Resistencia } \\
400 \mathrm{~m}\end{array}$ & $\mathbf{N}$ & $\mathrm{C}$ \\
\hline 1 & 8 & 11 & 16 & $\mathrm{NO}$ & 14 & 20 & $\mathrm{NO}$ & $2: 21$ & $2: 13$ & $\mathrm{NO}$ \\
\hline 2 & 8 & 10 & 16 & NO & 12 & 20 & NO & $2: 25$ & $2: 13$ & NO \\
\hline 3 & 8 & 9 & 16 & $\mathrm{NO}$ & 13 & 20 & NO & $2: 35$ & $2: 13$ & NO \\
\hline 4 & 8 & 11 & 16 & $\mathrm{NO}$ & 15 & 20 & NO & $2: 11$ & $2: 13$ & SI \\
\hline 5 & 8 & 13 & 16 & $\mathrm{NO}$ & 12 & 20 & $\mathrm{NO}$ & $1: 59$ & $2: 13$ & SI \\
\hline 6 & 9 & 18 & 17 & SI & 15 & 22 & NO & $2: 25$ & $2: 10$ & NO \\
\hline 7 & 9 & 16 & 17 & $\mathrm{NO}$ & 17 & 22 & NO & $2: 30$ & $2: 10$ & $\mathrm{NO}$ \\
\hline 8 & 9 & 19 & 17 & SI & 17 & 22 & NO & $1: 57$ & $2: 10$ & SI \\
\hline 9 & 9 & 19 & 17 & SI & 19 & 22 & NO & $2: 02$ & $2: 10$ & SI \\
\hline 10 & 9 & 20 & 17 & SI & 18 & 22 & NO & $2: 25$ & $2: 10$ & NO \\
\hline 11 & 9 & 17 & 17 & SI & 22 & 22 & NO & $2: 21$ & $2: 10$ & NO \\
\hline 12 & 9 & 18 & 17 & SI & 20 & 22 & NO & $2: 11$ & 2:10 & $\mathrm{NO}$ \\
\hline 13 & 9 & 19 & 17 & SI & 18 & 22 & NO & $2: 21$ & $2: 10$ & $\mathrm{NO}$ \\
\hline 14 & 10 & 21 & 17 & SI & 20 & 23 & NO & $2: 35$ & 2:03 & $\mathrm{NO}$ \\
\hline 15 & 10 & 20 & 17 & SI & 23 & 23 & SI & $2: 16$ & 2:03 & NO \\
\hline 16 & 10 & 21 & 17 & SI & 24 & 23 & SI & 2:01 & 2:03 & SI \\
\hline 17 & 10 & 15 & 17 & NO & 21 & 23 & NO & $2: 13$ & 2:03 & $\mathrm{NO}$ \\
\hline 18 & 10 & 14 & 17 & $\mathrm{NO}$ & 21 & 23 & NO & $2: 11$ & 2:03 & NO \\
\hline 19 & 10 & 18 & 17 & SI & 25 & 23 & SI & $2: 11$ & 2:03 & $\mathrm{NO}$ \\
\hline 20 & 10 & 19 & 17 & SI & 25 & 23 & SI & $2: 12$ & 2:03 & $\mathrm{NO}$ \\
\hline 21 & 10 & 15 & 17 & $\mathrm{NO}$ & 19 & 23 & NO & $2: 04$ & 2:03 & $\mathrm{NO}$ \\
\hline 22 & 10 & 13 & 17 & $\mathrm{NO}$ & 21 & 23 & NO & $2: 03$ & 2:03 & SI \\
\hline 23 & 10 & 16 & 17 & $\mathrm{NO}$ & 22 & 23 & NO & $2: 05$ & 2:03 & NO \\
\hline 24 & 11 & 16 & 18 & $\mathrm{NO}$ & 20 & 26 & NO & 2:01 & 2:03 & SI \\
\hline 25 & 11 & 14 & 18 & NO & 25 & 26 & NO & $1: 59$ & 2:03 & SI \\
\hline 26 & 11 & 17 & 18 & NO & 24 & 26 & NO & $1: 55$ & 2:03 & SI \\
\hline 27 & 11 & 18 & 18 & SI & 27 & 26 & SI & 2:09 & 2:03 & $\mathrm{NO}$ \\
\hline 28 & 11 & 22 & 18 & SI & 28 & 26 & SI & $2: 11$ & 2:03 & NO \\
\hline 29 & 11 & 16 & 18 & $\mathrm{NO}$ & 24 & 26 & NO & $2: 21$ & 2:03 & $\mathrm{NO}$ \\
\hline 30 & 11 & 22 & 18 & SI & 29 & 26 & SI & $2: 02$ & 2:03 & SI \\
\hline $\mathbf{T}$ & 9,63 & 16,57 & & $50,00 \%$ & 20,33 & & $23,33 \%$ & $2: 12$ & & $33,33 \%$ \\
\hline
\end{tabular}

En los tres sujetos estudiados, la tabla 16 especifico las pruebas de Planchas o Lagartijas, la Prueba de Abdominales (Abd) y la Prueba de Resistencia en 400m, en conjunto con las 
normativas o baremos $(\mathrm{N})$ establecidas al detalles en el próximo párrafo, así como el cumplimiento o no del mismo (C). Las medias del test de las Planchas se establecieron en 16,57 movimientos para el tiempo establecido, cumplimentando solamente el $50 \%$ de la muestra (15 sujetos), siendo este el valor más alto cumplimentado por las atletas en comparación con el resto de las pruebas de valoración del rendimiento. Para el caso de la prueba de los abdominales, la media se estableció en 20,33 movimientos, cumplimentado exitosamente el test el 23,33\% (7 sujetos) de la muestra, y para la prueba de valoración concerniente al test de la resistencia en $400 \mathrm{~m}$ la media se estableció en 2:12min, cumplimentado la prueba solamente el 33,33\% de la muestra (10 sujetos).

Para el caso de las tres pruebas mencionadas con anterioridad los baremos establecidos fueron:

a) La cantidad de planchas o lagartijas establecidas para el primer nivel por rango de edad es el siguiente:

1) 8 años: 16 movimientos

2) 9 y 10 años: 17 movimientos

3) 11 años: 18 movimientos.

b) La cantidad normada de abdominales (Abd) establecidos para el primer nivel por rango de edad son las siguientes:

1) 8 años: 20 movimientos

2) 9 años: 22 movimientos

3) 10 años: 23 movimientos

4) 11 años: 26 movimientos

c) Los tiempos en minutos establecidos para el primer nivel de resistencia $(400 \mathrm{~m})$ por rango de edad son los siguientes:

1) 8 años: $2: 13 \mathrm{~min}$

2) 9 años: $2: 10 \mathrm{~min}$

3) 10 y 11 años: 2:03min

En la figura 1 se evidencia el esquema general para el diseño de las acciones estratégicas para disminuir la deserción deportiva en el atletismo femenino de la Federación Deportiva de Loja. 


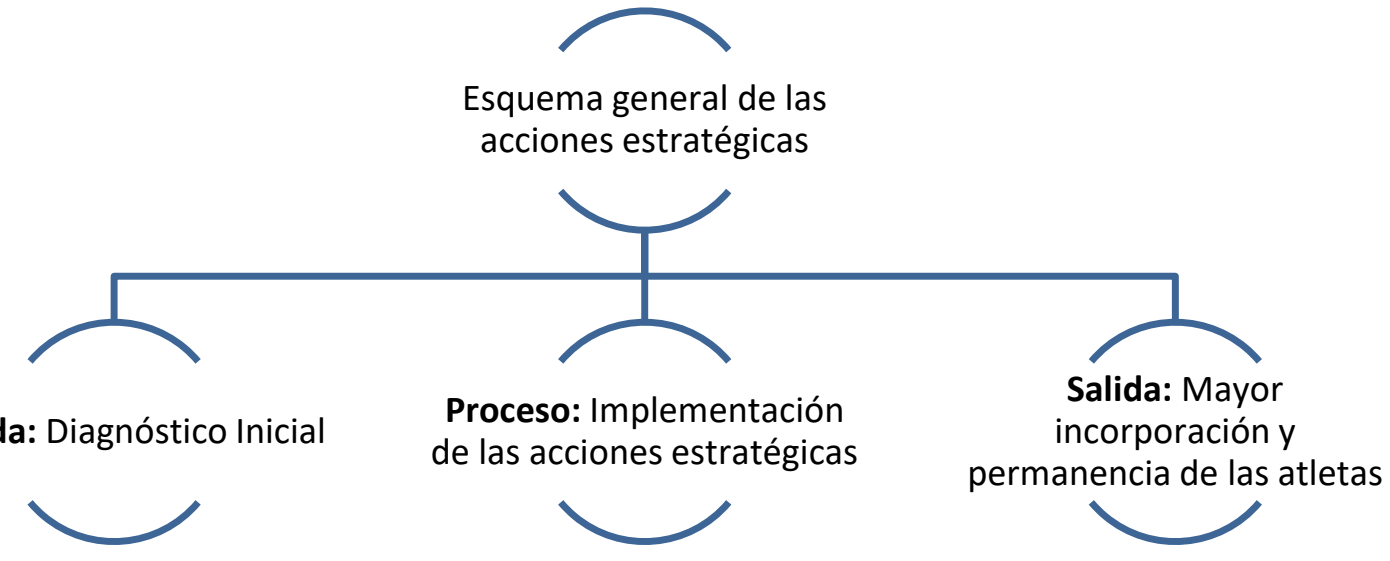

Figura 1: esquema general para el diseño de las acciones estratégicas para disminuir la deserción deportiva

El Grupo de acciones para incrementar la captación de deportistas del sexo femenino en el atletismo y la permanencia en los entrenamientos se evidencia a continuación:

1) Coordinación con las organizaciones escolares y familiares de la comunidad, para de conjunto establecer acciones que permitan garantizar la ejecución de actividades deportivas recreativas en la comunidad, cuyo propósito promueva la masividad del deporte desde el barrio y las escuelas; lo que posibilita estimular la participación de las niñas y de los padres con vista a incrementar la incorporación al deporte del Atletismo.

2) Divulgación de las actividades deportivas recreativas en saludos a diferentes fechas a nivel comunitario y cantonal, así como crear las condiciones adecuadas para garantizar la ejecución de todas las actividades incluidas tales como: Carrera de 30 m y de $400 \mathrm{~m}$, salto largo sin carrera de impulso, lanzamiento de la pelota, relevo, y el equilibrio, además de incluir juegos deportivos colectivos e individuales como el Voleibol, Ajedrez, Saltos en saco como parte de la recreación activa; baile, entre otros juegos que deseen las niñas(os). 
3) Establecer vínculo de trabajo desde el comienzo del curso con los profesores de Educación Física de las escuelas de la comunidad y realizar convocatoria para que las niñas/os participen en las actividades deportivas recreativas comunitaria y además dar a conocer de la existencia de un área deportiva encargada de impartir deportes en la localidad.

4) Visitas a las escuelas con vista a divulgar la existencia de una propuesta de un proyecto que promueve la incorporación de las niñas al Atletismo desde edades tempranas.

5) Vincular al Ministerio del Deporte y otros ministerios en el apoyo metodológico y científico-técnico, así como económico, a los proyectos comunitarios relaciones con la selección deportiva en el país.

6) Participar como observador en todas las pruebas de Eficiencia Física realizadas en las escuelas de la comunidad de Loja, lo que permite desde ese mismo momento comenzar el trabajo de captación de las niñas que deseen incorporarse al Atletismo, aun cuando el resultado de las pruebas no sean sobresalientes, pues de la masividad surgen los verdaderos talentos.

7) Las niñas captadas al concluir las pruebas de Eficiencia Física, deben ser convocadas para que durante los meses de mayo y junio realicen sus actividades iníciales basada fundamentalmente en juegos, introducción de elementos técnicos y flexibilidad, además es el momento de comenzar a familiarizarse en el grupo, al concluir esos dos meses se realiza una prueba de Eficiencia Física para evaluar el nivel inicial real con el cual comienzan las niñas; lo que permite al entrenador trazar objetivos específicos dirigidos a desarrollar en las niñas los elementos de interés o motivación para lograr los objetivos del programa establecido para la categoría infantil en términos de masividad desde las edades tempranas en el Atletismo.

8) Informar en las escuelas los resultados relevantes obtenidos por las niñas en las competencias locales, provinciales y nacionales; aspecto que la motiva a permanecer en el Atletismo y además puede estimular la reincorporación de las que abandonaron la práctica deportiva especializada, así como de las nuevas incorporaciones. 
9) Entrega de certificados en las actividades deportivas recreativas realizadas en la comunidad; lo que alguna manera motiva la participación; así como meriendas en las medidas de las posibilidades.

10) Mantener a los padres debidamente informado sobre la asimilación de la niña en relación al programa de enseñanza y de los resultados obtenidos.

11) Establecer un sistema de comunicación permanente entre el entrenador y la familia; lo que permite la cooperación mutua entre ambos, en aras de alcanzar los objetivos trazados en el programa.

12) Capacitar al claustro de profesores deportivos en el empleo de metodologías modernas de entrenamiento para la iniciación, con énfasis en la motivación del alumnado hacia la práctica de actividad física especializada, como es el caso de las diversas modalidades del atletismo.

13) Realizar actividades recreativas culturales en saludos fechas históricas de Loja y el país, estimulando la participación de las niñas y sus familiares, así como del profesorado.

14) Controlar por parte del departamento técnico de Federación Deportiva de Loja, los procesos metodológicos aplicados por los entrenadores de las niñas, evitando la violación de las etapas normales del desarrollo deportivo en edades tempranas.

15) Adaptar un sistema competitivo de acuerdo a las características y necesidades de las niñas de la comunidad Lojana, de tal manera que facilite la interacción e inserción en el sistema competitivo Nacional.

16) Facilitar la implementación necesaria y complementaria a los entrenadores, de tal manera que se les facilite la variación de las actividades a ejecutar en el programa de entrenamiento, y así evitar que se genere un estrés psíquico por parte de las deportistas, y por ende se evita la deserción deportiva.

17) Crear proyectos para generación de recursos económicos, donde se incluyan a los padres de familia, de tal manera que facilite la participación de las niñas en eventos que no cuente con el financiamiento económico de la Federación Deportiva de Loja.

18) Organizar actividades lúdicas, donde interactúen padres de familia, entrenadores y deportistas, de tal manera que permita fomentar la unión y camaradería entre todos los actores del atletismo femenino infantil en Loja• 
19) Fomentar los valores de la competición como una fuente importante de motivación recreación y aprendizaje, evitando caer en un factor de estrés y presión de resultados tanto de las deportistas como de los padres de familia.

Crear una guía de control académico de las niñas, controlándolas y orientándolas en sus programas de estudios, evitando que las deportistas bajen su rendimiento escolar a causa del tiempo empleado en el entrenamiento deportivo.

\section{Conclusiones.}

- Las diversas fuentes de investigación consultadas, evidenció la necesidad de establecer estrategias eficientes en la modelación del entrenamiento deportivo para la iniciación del atletismo, enfatizando en los aspectos de la selección y permanencia deportiva, así como de la influencia de la familia en el apoyo a la niña deportista.

- Se determinó algunas variables de interés en las alumnas y sus familiares, las cuales sirvieron para el diseño de las acciones estratégicas.

- Los diversos test de valoración del rendimiento deportivo empleados, determinaron el nivel inicial del rendimiento individual de cada sujeto estudiado, aspecto que facilitará potenciar la modelación futura del entrenamiento, adaptando los contenidos de la preparación a las necesidades actuales del deportista, e implementando las acciones pertinentes para la permanencia del sujeto y la potenciación de sus resultados deportivos.

- Se conformó las acciones estratégicas para incrementar la captación de deportistas del sexo femenino en el atletismo y la permanencia en los entrenamientos, permitiendo mantener la comunicación entre la familia, las niñas, el profesorado y los entrenadores.

\section{Referencias bibliográficas.}

Beattie, K., Kenny, I. C., Lyons, M., \& Carson, B. (2014). The effect of strength training on performance in endurance athletes. Sports Medicine, 44(6), 845-865.

Bompa, T. O. (2005). Entrenamiento para jóvenes deportistas (1 ed.). Barcelona: Editorial Hispano Europea. 
Camino, J. R., \& Ayala, V. M. (2012). Marketing y fútbol: el mercado de las pasiones (1 ed.). Madrid: Esic Editorial.

Chávez Hernández, J. P. (2015). Estudio de los factores que inciden en la deserción de los deportistas en la Escuela Superior Politécnica del Chimborazo 2012, Propuesta alternativa (Master's thesis, Universidad de las Fuerzas Armadas ESPE. Máster, Universidad de las Fuerzas Armadas ESPE, Ciencias Humanas y Sociales, Quito.

Chávez, J. P., \& Calero, S. (Junio de 2015). Factores físicos, socio-económicos y psicológicos que inciden en la deserción deportiva en la Escuela Superior Politécnica de Chimborazo. In Congreso de Ciencia y Tecnología. Lecturas: educación física y deportes, 20(211), 1-9.

Chelladurai, P., \& Kerwin, S. (2017). Human resource management in sport and recreation (3 ed.). Human Kinetics.

Del Coso, J., Areces, F., Salinero, J. J., González-Millán, C., Abián-Vicén, J., Soriano, L., \& Calleja-Gonzalez, J. (2014). Del Coso, J., Areces, F., Salinero, J. J., González-Millán, C., Abián-Vicén, J., Soriano, L.,Compression stockings do not improve muscular performance during a half-ironman triathlon race. European journal of applied physiology, 114(3), 587-595.

Ernesto Avella, R., \& Medellín, J. P. (2013). Perfil dermatoglífico y somatotípico de atletas de la selección colombia de atletismo (velocidad) participante en los juegos panamericanos de Guadalajara, 2011. Revista UDCA Actualidad \& Divulgación Cientifica, 16(1), 17-25.

Espinoza Loja, M. Y. (2009). Entrenamiento deportivo para atletas juveniles de pruebas de velocidad y salto de longitud. Bachelor's, Universidad de Cuenca, Facultad de Filosofía, Letras y Ciencias de la Educación, Cuenca.

Gamble, P. (2013). Strength and conditioning for team sports: sport-specific physical preparation for high performance (2 ed.). Routledge. 
Granell, J. C., \& Lazcorreta, J. E. (2004). LAS TÉCNICAS DE ATLETISMO. Manual práctico de enseñanza (1 ed.). Barcelona: Editorial Paidotribo.

Knudson, D. V. (2013). Qualitative diagnosis of human movement: improving performance in sport and exercise ( 3 ed.). Human kinetics.

López-Walle, J., Tristán,, J., Cantú-Berrueto, A., Zamarripa, J., \& Cocca, A. (2013). Propiedades psicométricas de la Escala de Frustración de las Necesidades Básicas en el Deporte. Revista Mexicana de Psicología, 30, S2038-S2041.

Marín, C., \& Eduardo, G. (2013). La ansiedad en el rendimiento de los deportistas de atletismo en la etapa pre-competitiva y competitiva, la Federación Deportiva de El Oro, periodo 2012-2013. Bachelor's, Universidad Técnica de Machala, Carrera de Psicologia Clínica, Machala.

Martin, D. (1993). Entrenamiento multilateral y especialización precoz. Stadium, 27(160), $36-42$.

Martin, D., \& Nicolaus, J. (2004). Metodología general del entrenamiento infantil y juvenil (1 ed., Vol. 24). Barcelona: Editorial Paidotribo.

McGannon, K. R., \& Smith, B. (2015). Centralizing culture in cultural sport psychology research: The potential of narrative inquiry and discursive psychology. Psychology of Sport and Exercise, 17, 79-87.

Morales, V., \& Priscila, E. (2016). Estrategias de promoción para deportistas de alto rendimiento en la disciplina de Atletismo de la ciudad de Quito. Licenciatura, Universidad de Los Hemisferios, Ciencias Empresariales, Quito.

Núñez, U. C. (2000). La iniciación a los deportes desde su estructura y dinámica: Aplicación a la Educación Física Escolar y el Entrenamiento Deportivo (1 ed., Vol. 504). Barcelona: Inde.

Pico Medina, D. E., \& Brazales Cervantes, D. A. (2014). Incidencia de la frecuencia cardíaca y el lactato en el rendimiento físico de los atletas del equipo élite de 
orientación del ejército ecuatoriano en el año 2012. Bachelor's, Universidad de las Fuerzas Armadas ESPE, Ciencias Humanas y Sociales, Quito.

Sanjurjo, J. A. (2012). Conquistando a las masas: el impacto del deporte en la prensa española, 1900-1936. Recorde: Revista de História do Esporte, 1, 1-40.

Seners, P. (2001). Didáctica del atletismo (1 ed.). Barcelona: Inde.

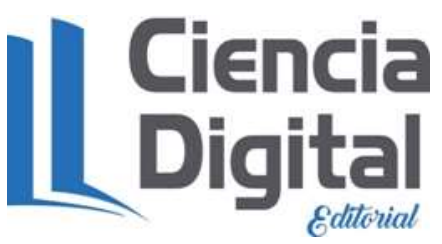




\section{Para citar el artículo indexado.}

Montoro R., Quizhpe V., Ochoa E. \& Valverde L. (2018). Valoración del rendimiento físico inicial y acciones estratégicas para disminuir la deserción deportiva en el atletismo femenino de la Federación Deportiva de Loja. Revista electrónica Ciencia Digital 2(4), 6-33. Recuperado desde:

$\underline{\text { http://cienciadigital.org/revistacienciadigital2/index.php/CienciaDigital/article/view/206/19 }}$ $\underline{3}$

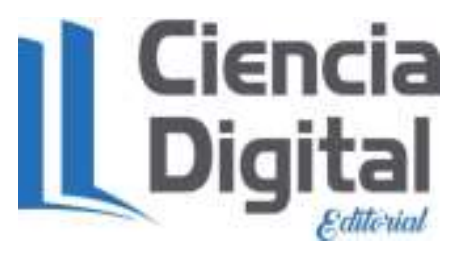

El artículo que se publica es de exclusiva responsabilidad de los autores y no necesariamente reflejan el pensamiento de la Revista Ciencia Digital.

El articulo queda en propiedad de la revista y, por tanto, su publicación parcial y/o total en otro medio tiene que ser autorizado por el director de la Revista Ciencia Digital.
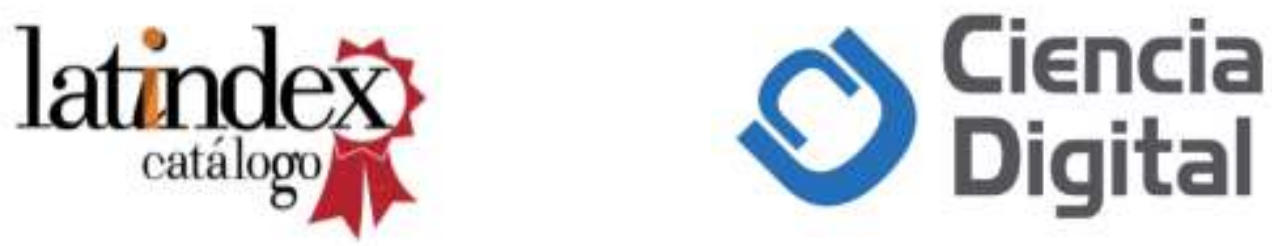